(C) 2016, Elsevier. Licensed under the Creative Commons Attribution-NonCommercial-NoDerivatives 4.0 International

http://creativecommons.org/licenses/by-nc-nd/4.0/

\title{
Putting children forward for epilepsy surgery: A qualitative study of UK parents' and health professionals' decision-making experiences.
}

\author{
Authors: Gemma Heath ${ }^{1,2^{*}}$, Shanara Abdin ${ }^{1}$, Rahima Begum ${ }^{1} \&$ Shauna Kearney ${ }^{2}$ \\ ${ }^{1}$ Department of Psychology, Aston University, Birmingham, UK \\ ${ }^{2}$ Department of Psychology, Birmingham Children's Hospital, Birmingham, UK
}

*Correspondence: E-Mail: g.heath1@aston.ac.uk; Tel.: +44-0121-204-4008

\begin{abstract}
Background: Against a backdrop of recommendations for increasing access to and uptake of early surgical intervention for children with medically intractable epilepsy, it is important to understand how parents and professionals decide to put children forward for epilepsy surgery and what their decisional support needs are.

Aim: To explore how parents and health professionals make decisions regarding putting children forward for paediatric epilepsy surgery.

Methods: Individual interviews were conducted with nine parents of children who had undergone paediatric epilepsy surgery at a specialist children's hospital, and ten healthcare professionals who made-up the children's epilepsy surgery service multi-disciplinary healthcare team (MDT). Three MDT meetings were also observed. Data were analysed thematically.
\end{abstract}

Findings: Four themes were generated from analysis of interviews with parents: Presentation of surgery as a treatment option; Decision-making; Looking back; Interventions. Three themes were generated from analysis of interviews/observations with health professionals: Triangulating information; Team working; Patient and family perspectives.

Discussion: Parents wanted more information and support in deciding to put their child forward for epilepsy surgery. They attempted to balance the potential benefits of surgery against any risks of harm. For health professionals, a multidisciplinary approach was seen as crucial to the decisionmaking process. Advocating for the family was perceived to be the responsibility of non-medical professionals.

Conclusion: Decision-making can be supported by incorporating families into discussions regarding epilepsy surgery as a potential treatment option earlier in the process, and by providing families with additional information and access to other parents with similar experiences.

Keywords: Qualitative Research; Epilepsy; Decision-making; Parents; Healthcare Professionals 


\section{Putting children forward for epilepsy surgery: A qualitative study of UK parents' and health professionals' decision-making experiences.}

\section{Background}

For children with prolonged, medically intractable epilepsy, surgical intervention can lead to improvements in quality of life by reducing the number and severity of seizures [1-3] and slowing the rate of cognitive decline. Moreover, evidence relating to the negative impact that ongoing seizures have on brain development have led to calls for consideration of surgery as a treatment option at an earlier age (under 5 years) [4]. However, when examining the activity for pediatric epilepsy surgery in England against international benchmarks, less than half the numbers of procedures are performed each year that would be considered beneficial to this population [4]. While it is unclear exactly why UK referral rates for paediatric epilepsy surgery are low in comparison to other countries, possible explanations might include attitudinal differences towards surgical intervention in children, funding differences or differences in clinical practice [5].

Despite justification of surgical treatment for children with uncontrolled epilepsy in terms of medical and psycho-social burden, there may also be risks attached, for example, impairments to memory or thinking skills [6]. Thus, in cases where surgery is a viable option, families face difficult treatment decisions. The shared decision-making model used in paediatric healthcare proposes that decisions regarding treatment are shared between a team of health professionals, parents and patients (where appropriate). How these decisions are arrived at is less clear.

From a parent perspective, research identifies several factors influencing treatment decision-making including parents' emotional responses; balancing the child's interests with their own; information from, and trust in professionals; uncertainty regarding outcomes; and pressures from people or time [7]. Limited research has specifically explored parental decision-making in relation to paediatric epilepsy surgery. A recent systematic review [8] identified nine studies examining patient perceptions of epilepsy surgery, of which, only two related to parents' views [1, 2]. A further study has since reported the perspective of parents of children who have undergone epilepsy surgery [9]. These papers suggest that parental decision-making is influenced by the patient's age, parental knowledge of the condition and their treatment perceptions. Moreover, given variability in each child's decision-making capabilities, parents can struggle to know when and how to include their child [10].

From a clinical perspective, each designated Children's Epilepsy Surgery Service (CESS) in England has a clinical pathway that encompasses a specialist multi-disciplinary healthcare team (MDT). Teams are constituted according to national guidance (4). Key members include surgeons, neurologists, neuropsychologists and the therapies. A range of neuro-radiological investigations and neurophysiological findings are considered, as well as the clinical presentation of the child/young person. Treatment options are discussed within this context, including the potential risks and benefits of surgery. MDT decisions are then shared with the family and a plan agreed.

The few studies that have explored professionals' views regarding epilepsy surgery $[3,11,12]$ report variable attitudes, indicating an underlying uncertainty or ambivalence towards this treatment [11]. Barriers to epilepsy surgery referral include insufficient knowledge about the procedure and outcomes, and lack of clarity regarding the concept of refractory epilepsy and drug resistance. Such 
shortfalls have led to delays in referring patients for surgical intervention and overextension of suboptimal pharmacological treatments [11]. While contributing valuable insights, these studies do not consider how MDTs agree to propose a child for epilepsy surgery. Research examining influences on MDT decision-making within the context of other health services reveal that professional expertise, government health policy, and familiarity with the patient are key factors in shaping treatment decision-making $[13,14]$.

Against a backdrop of recommendations for increasing access to and uptake of epilepsy surgery in those children for whom surgical intervention is an option and would be of benefit [4], it is imperative that we improve understanding of how parents and professionals make decisions regarding paediatric epilepsy surgery, in order to be able to address their decisional support needs.

\section{Aim}

To explore how parents and health professionals make decisions regarding paediatric epilepsy surgery.

\subsection{Objectives}

- To identify factors that influence the process of decision-making regarding paediatric epilepsy surgery from the perspective of parents and professionals.

- To identify the support needs of those considering surgery as a treatment option for a child with medically intractable epilepsy.

\section{Method}

\subsection{Design}

As the aim was to explore, in-depth, the processes of decision-making from the perspectives of those making the decisions, the study employed a qualitative methodology using semi-structured interviews and non-participant observations. A favourable review was obtained from an NHS Research Ethics Committee prior to commencement of the study (REC Ref: 15/WM/0123).

\subsection{Setting}

The study was undertaken at a UK specialist children's hospital which hosts one of four national centres in England, commissioned to provide a Children's Epilepsy Surgery Service (CESS).

\subsection{Sample}

Purposive sampling was used to generate a diverse sample of parents who had put their child forward for epilepsy surgery and healthcare professionals who made-up the CESS multi-disciplinary healthcare team.

\subsection{Recruitment}

Parents were identified and recruited through a Nurse Specialist, who introduced them to the study by providing an invitation letter and information leaflet. Interested parents were then invited to contact the research team to discuss their participation and schedule a time for interview. Access to 
MDT meetings and identification of health professionals was facilitated by one the study investigators (SK) who was also a member of the paediatric epilepsy surgery MDT. Potential participants were recruited for interview via email invitation and information sheet.

\subsection{Data collection}

3.5.1 Observations: A member of the research team (RB) attended three MDT meetings over a twomonth period to observe how the process of decision-making was carried out. Detailed field notes were made using a data collection tool developed in accordance with best practice guidelines [15]. This focused on the process of team decision-making, including how MDT members interacted and arrived at a consensus agreement, what function or role each MDT member played and what their contributions were. Informed consent was obtained prior to data collection.

3.5.2 Interviews: Parents and health professionals participated in individual interviews which were digitally audio-recorded. To promote inclusivity, a choice of interview methods was offered (e.g. face-to-face, telephone, Skype) at times/locations of the participant's choice (e.g. home, hospital). Interviews were guided by a semi-structured schedule of open questions based on concepts identified from the literature and for professionals, on observations of the MDT meetings. Parent interviews explored how they experienced decision-making regarding their child's epilepsy surgery, motivations and beliefs that influenced their decisions and resources they found helpful or would be helpful for others. Health professional interviews explored their experience of deciding that a child was eligible for surgery, their role in this process and views on how decision-making could be improved. Interviews were facilitated by a member of the research team (SA, RB) and lasted approximately 1 hour.

\subsection{Data Analysis}

Data were transcribed verbatim, anonymised and analysed using an inductive thematic approach. Thematic Analysis is a flexible, systematic and transparent method that serves to highlight similarities and differences across the data set and facilitates the production of rich and interpretive, yet data-driven themes [16]. Analysis involved identifying and reporting patterns within the data through a process of familiarisation and coding before generating, defining and naming themes. Data were coded independently by three researchers (GH, SA, RB), who met regularly to discuss/refine emerging analyses.

\section{Findings}

\subsection{Participants}

Interview participants consisted of nine parents $(\mathrm{P})$ and ten health professionals. To maintain anonymity, health professionals were categorised either as Medical Professionals (MP) or Allied Health Professionals (AHP). See table 1.

\subsection{Findings from interviews with parents}

\subsubsection{Presentation of surgery as a treatment option}

Parents were clear that putting their child forward for epilepsy surgery was the best and right decision for their child, demonstrating a high level of satisfaction with the treatment outcome. 
However, initial perceptions of surgery were often negative, particularly if it was not a treatment option that had previously been considered or that professionals had indicated. Many parents described attending their child's clinic appointment expecting to talk about more conventional treatments (e.g. medication or diet), leading to them feeling surprised when surgery was raised for discussion.

"We went originally to talk about the ketogenic diet and then the doctor said 'oh I think surgery's your best option' which came as a bit of a shock really because no one had ever mentioned the surgery before." (P5)

"It actually came as a bit of a surprise to us that option, in that it was mentioned in a review visit, that maybe the time had come to look at the option of surgery but we weren't really aware of that option at that point so it came as quite a shock to us." (P6)

While some parents took comfort from the possibility of an additional treatment pathway, others felt excluded from the decision to assess their child for surgical candidacy, leading to their feeling unable to participate in an informed discussion.

\subsubsection{Decision-making}

Following indication of surgery as a potential treatment, parents and families faced what they described as a "difficult decision" (P5) which was experienced as "daunting", "frightening", "scary" and "stressful" (P 1, 4, 5, 6, 7, 9). However, they also reported a sense of duty to be "strong" (P1) and "brave" $(P 3,4)$ for their children, wishing to protect them from their feelings of anxiety.

\subsubsection{Balancing potential costs and benefits}

When deliberating surgery, parents balanced their perceived necessity of the treatment in terms of how epilepsy currently affected and would, in the future, affect their child's quality of life, against concerns regarding the possibility of negative outcomes. In other words, parents weighed-up the potential benefits of surgery such as freedom from seizures against what they understood as the risks, including cognitive impairments.

"There was no quality of life for him; we had to do what was best. We were told of the risks of the surgery and we were told that actually this would be the best thing for him... we don't regret it, but it was, it's not an easy decision to take because you're worried about the risks of things." (P2)

Such a decision was perceived to carry a high degree of responsibility for parents, which was deemed burdensome. Parents discussed feeling pressure from a need to 'do the right thing' by their child. That is, choosing the treatment option that would give their child the best chance of reaching their full potential. For all parents in this study, the perceived need of surgery outweighed the possible risks and side effects.

"We thought this is going to be no life for him... He was on this diet which meant he couldn't go out with friends; he couldn't stay at friends' houses, all the things that other kids do, he couldn't do... and then he got these fits virtually every night and we thought... you know 
what, there is no decision to make, we gotta do it... We knew there were risks, we talked about them with [child] and he was frightened but he said no, we gotta do it." (P3)

Some parents were unconvinced about the efficacy of surgery for reducing their child's seizures. Scepticism stemmed from their experience of other pharmacological and dietary treatments which had resulted in little improvement in seizure control. In weighing-up treatment options, parents also discussed the side-effects of alternative treatments. For example, medications were perceived to have a range of adverse side-effects, whereas ketogenic diets were viewed as negatively affecting the child's ability to live a 'normal' life.

"We thought about the effects of the medication if we chose to continue on the path of finding a different medication... but then how long would that have taken us? Like how long would it work for? Then it would develop, then how long 'til we have to find something else, with all the side-effects that can happen? I didn't want to put her through that as well." (P9)

\subsubsection{In search of information}

Parents perceived the information they received from professionals as inadequate to make an informed decision. They therefore sought out additional information regarding the surgical procedure and outcomes. Aiming to comprehend what surgery would entail, parents requested information in lay language, and searched the internet for information and videos. Increasing their own knowledge about medically intractable epilepsy and the available treatment options empowered parents to participate more fully in the decision-making process.

"I said to him 'don't tell me in a doctor's term, tell it me in a parent's term. I want to know the ins and outs; I want to know exactly what the risks are but in terms that I understand'." (P4)

"I googled it until I couldn't google anymore and I think that gave me a better understanding of what was going on." (P7)

\subsubsection{Family decisions}

Parents described decision-making as a family choice involving partners, siblings, grandparents and the child (where developmentally appropriate). Discussing treatment options with family members was generally considered to be helpful.

"I remember my mother-in-law was in Pakistan and I called her up and I was very teary... I felt like I had been punched in the stomach to be honest 'cause, you know, brain surgery is not something you take lightly and she said 'well if it's for his betterment and to improve his life then we will do whatever it takes'." (P2)

Although for some families it did give rise to conflicts. For example, one Mother described herself as more agreeable to surgery than the child's Father because, as the primary carer, she 'witnessed' the true extent of their child's seizures. In cases where the child's epilepsy was considered to impact on the whole family (e.g. where a parent could not return to work because of a need to look after the child), then the decision was understood as a whole family responsibility.

\subsubsection{Last treatment of choice}


For most parents, surgery was a last resort treatment option that would only be considered following the exhaustion of all other options. This perception was influenced by parental fear regarding the irreversibility of surgery, as well as by professionals who had informed them that surgery was the only remaining treatment for controlling their child's seizures. Some parents felt they had little alternative but to accept surgical intervention in order to provide their child with the best possible chance of improving their quality of life.

"We felt it was our only option really in her having a more independent future." (P5)

"My husband and I walked out of the appointment saying we feel like we don't have a choice... we felt like it was the only choice we could make." (P9)

For one family with an adolescent child, it was considered necessary to accept surgery while the child received care from the Children's Hospital as they believed that the treatment would become unavailable once transitioned to adult services. When a decision to accept surgery had been made, parents hoped for the procedure to be carried out as soon as possible. Waiting for a date for surgery was described as an anxious time for parents, who experienced relief when a date was set.

\subsubsection{Looking back}

On reflection, all parents were satisfied with their treatment choice and had no regrets about the decision to accept surgical intervention. Parents considered surgery to be a good experience which produced positive outcomes for their child, despite some continuing to experience symptoms or complications (e.g. seizures following treatment, minor memory loss).

"It's the best thing we've ever done for our son, it was the best thing, we don't regret it, we don't regret the decision at all... it has given him a whole new lease of life." (P2)

"Now surgery is over, I know I did the right thing... at the moment I can say it's one of the best decisions I've made in my life." (P4)

\subsubsection{Interventions}

A number of strategies were recommended for helping to meet parents' decisional support needs. These included earlier and softer introduction of surgery as a possible treatment option, additional information provision and peer support. It became apparent that parents perceived a lack of control over the decision-making process through their limited involvement in initial discussions regarding surgical candidacy. Empowering parents by involving them in decision-making from the outset was recommended to help them feel incorporated, rather than consulted once a decision had been made.

"It would have helped us if [surgery] had been mentioned in previous years as an option 'cause it hadn't been mentioned as an option. It did come out of the blue... I think it probably would have helped maybe if there was a little bit more acknowledgement of how big a deal it is for parents." (P6)

In addition, parents emphasised the importance of accessing the experience and expertise of other parents who had followed a similar treatment pathway. Such peer support was suggested to 
facilitate understanding of the surgical procedure from a 'parental perspective', as well as provide emotional support.

"If people could freely put their experiences on either a forum or even if you get a group, you know, parents talking to other parents who have gone through the experience to get a real idea of what's happening." (P8)

Finally, parents emphasised the importance of receiving adequate information regarding epilepsy surgery, including information presented in a variety of formats (e.g. booklets, photographs, videos). They also suggested that a 'Frequently Asked Questions' information sheet would have been beneficial.

"I know I got a little booklet about epilepsy surgery services, but it would have been nice for us to have some extra information... just some written up case studies of kids that have gone through, had the same, you know, operation." (P5)

\subsection{Findings from interviews and observations with health professionals}

\subsubsection{Triangulating information}

Information presented at MDT meetings was structured by a PowerPoint presentation. This facilitated decision-making by offering a systematic and efficient mode of communication which captured different aspects of the child's condition and indicated points for discussion. It summarised the child's status, medical history, seizure type and current medication, and on occasion, patientrelated factors (e.g. child's behaviour, parental concerns). Representatives from multiple disciplines then presented the outcome of their assessments.

"We have the PowerPoint that takes us through in an orderly manner... it is pretty structured and I think we all have a clear methodical way compared to other MDTs. It is quite cohesive in a sense that there is a format." (AHP)

A need to justify the decision to put a child forward for epilepsy surgery became apparent from the importance placed on information gathered from the child's investigations. However, the information required for decision-making was not considered valid if it only came from one source. For instance, evidence from MRI scans had to be triangulated with other sources of information, such as EEG scans and information regarding the semiology of seizures. This triangulation facilitated a multi-perspective approach, which was thought to prevent biased decision-making.

"You are looking for concordance between the MRI scan and the EEG and the type of seizure they are having. That's the strategy we are following all the time and you want those to line up. In an ideal world, they all line up. In a non-ideal world, some of them line up and some of them don't and that's when obviously judgements come into, whether you feel that there is enough there to proceed with a surgical resection." (MP)

Team confidence in continuing on a surgical pathway was thus influenced by whether information gathered from a range of sources supported such action. Misalignments or uncertainties between information sources generated questions, resulting in requests for further investigations. This 
guarded approach reflected the irreversible nature of the treatment. Lack of confidence from any professional prevented a final decision from being made.

"It varies from very easy decisions where it hardly needs a multidisciplinary approach to much more complex decisions, where everybody needs to input and sometimes it's impossible to reach a decision and you're not going to go ahead with surgery generally speaking." (MP)

While professionals discussed the importance of eliciting and incorporating opinions and assessments from multiple disciplines, observations exposed the privileged nature of disease-related information, indicating a differential emphasis on medical evidence such as MRI or EEG scans. In this way, medical professionals were perceived to maintain a dominant role as the information they presented could be verified by objective 'factual' evidence.

"They [Medical Professionals] don't see therapy information as potentially as essential in decision-making, they would see the sort of neurophysiology, the neuropsychology assessment as key." (AHP)

This led some professionals to question how 'multi-disciplinary' decision-making was; indicating that in some cases, the decision to propose a child as a surgical candidate had been made prior to the MDT meeting:

"I am less clear about where that decision-making takes place, whether it is in the meeting or whether it is prior to that." (AHP)

At the end of each case presentation, a plan was made. If professionals could not agree, if the surgical procedure was deemed technically complicated or unsafe, or if a case was particularly complex, then either further investigations were requested, or the case was referred to a national CESS meeting, or the patient was removed from the epilepsy surgery programme.

\subsubsection{Team working}

When assessing a child for surgical candidacy the importance of working within a cohesive team, towards a common goal was emphasised. Moreover, bringing together different spheres of expertise to deliberate the case for each child was perceived to safeguard the patient, ensuring all factors were accounted for, before surgery was considered an option.

"We work as a team and every member of the team has a role, a role that is very very crucial and all these cogs have to be working together." (MP)

Being entrusted with the responsibility to decide if a child should be put forward for surgery was observed to be a difficult task. Nevertheless, professionals expressed that they were generally content with the process for making treatment decisions, which was favourably compared to that of other MDTs.

"The MDT is good I think... it proves to be very effective and there is always going to be challenges when you bring several discipline together, but actually on the whole we seem to work well and have produced good outputs." (MP) 
Despite decision-making being described as collaborative, the Neurosurgeon ultimately weighed-up all the information for each case and made a final decision. This role and responsibility was accepted by the team and justified in terms of it being "the surgeon who has to put his knife to a patient" (MP). Nevertheless, MDT members were clear that the Neurosurgeon could not make the decision for surgery in isolation. Other key professionals were perceived to include the Neurologist, the Neurophysiologist and Radiologist.

"Every member of the MDT contributes their opinion about whether surgery is a yes or a no, but ultimately the consultant decides based on the balance of everyone's opinion... but I don't think that is inappropriate." (AHP)

Contributions from AHPs were considered important in terms of providing a holistic overview of the child. However, in the same way that diverse types of information were treated differently, some AHPs felt that they struggled to make their assessment findings heard. This led to a feeling that their contributions were less valued than those of the 'core' medical team.

"It feels like sometimes you have to make your contribution rather than being invited to give a contribution." (AHP)

Despite this, professionals acknowledged how the team had evolved over time and how teamworking had improved since inception of the MDT:

"At the beginning, some of us had to fight more for our time, but now we are seen as part of the multidisciplinary team, so we will present our own views, so you feel more of an equal member of the team now, overtime." (AHP)

\subsubsection{Patient and family perspective}

Professionals were aware that families found the decision to opt for surgery difficult and that they placed a significant amount of trust in the team to act in their child's best interest. Liaising with, and advocating for the family was perceived to be the responsibility of AHPs, who were described as holding a key role in supporting parents throughout the process.

"We send a letter out straight away after the MDT as to what the decision is, however, sometimes the letters generate more questions than answers so that is why it is followed through with a phone call from the epilepsy nurse specialist." (MP)

It was clear that the MDT aimed to do what was beneficial for the child as soon as a referral to the epilepsy surgery programme was made. Professionals described feeling a sense of duty to protect families from unnecessary waiting and from "potential false hope" (MP). They were clear on the need to discontinue investigations once it became apparent that the child was "unlikely to ever benefit from epilepsy surgery" (MP).

Although the patient perspective was described as important, it was not systematically included in, or a predominant feature of the MDT meeting. Several professionals recognised that MDT meetings could be improved by incorporating patient-related information and that families could be included in the decision-making process more effectively: 
"It would be nice to have the child's picture if possible on there so it actually very much feels like it's very focused on the child... personal about a child rather than just clinical information." (AHP)

\section{Discussion}

This study explored the views and experiences of parents and health professionals on deciding to put a child forward for epilepsy surgery. Findings indicated that overall, families were satisfied with the service they received and had no regrets regarding their choice to accept surgery, describing it as the best and right decision for their child.

Nevertheless, parents' views regarding surgical intervention were perceived to be ascertained lateon in the decision-making process. This was justified by professionals in terms of their desire not to raise the families' hopes for a treatment option that might not be viable. Research suggests that inclination to accept surgery may depend on how and when the clinician chooses to raise and discuss the issue [1]. In our study, delays in presenting surgery as a potential treatment option led to some parents feeling excluded from decisions about their child's condition and treatment, leading to their feeling unable to participate in an informed discussion. Parents recommended that they be consulted on the range of treatments from the outset, with the understanding that not all options may be viable. This supports existing research suggesting that since, parents of younger children with epilepsy are more accepting of surgical intervention, there is little reason to delay consideration of this treatment option, in case the condition later becomes drug-resistant [1]. Similar findings have also been shown in related fields, such as oncology. A study by Kidger et al [13] found that while some professionals felt that patient views should not be sought until after the team had discussed possible treatment options, others argued that patient wishes should be taken into account at the point of MDT decision-making. Collaborative treatment planning between professionals and patients has been found to reduce negative perceptions of epilepsy surgery [17]. Parents of children with other medical conditions (e.g. cancer, congenital heart disease) have further been found to prefer collaborative decision-making over an autonomous or a paternalistic approach [10]

Also consistent with Kidger et al [12], our findings demonstrated that despite professionals acknowledging the importance of patient-related information in MDT decision-making, such information was rarely included in the process. We suggest that patient-related factors could be incorporated within MDT meetings by systematically including such information within case presentations. Allied Health Professionals would be ideally positioned to obtain and communicate this information. However, differing perceptions between professional groups regarding how their contributions are valued could hold implications for integrating the family perspective.

The perception of epilepsy surgery as frightening and a last resort treatment option reflects research from parental [9], patient [1, 17] and professional perspectives [11]. A study by Baca and colleagues [9] found parents to perceive epilepsy surgery as "pretty horrific" and a treatment of "last resort", describing the decision-making process as "scary". Similar to our findings, parents in this study also reported that their child tried many medications which failed to reduce seizures, before surgery was raised as a potential treatment option. Such delays in referral of children for epilepsy surgery have been reported elsewhere $[1,5,11]$. 
Our findings add that parents make judgments about their child's need for surgery in terms of the degree to which epilepsy impacts on their child's quality of life, balanced against concerns about possible adverse consequences of an irreversible treatment. This supports a recent systematic review of epilepsy surgery decision-making [8] which identified illness and treatment perceptions as central to the weighing of treatment risks and benefits. For parents, this decisional-balance exercise is experienced as both a cognitive and emotional process [18]. Research examining parent treatment choices in other paediatric conditions requiring surgery (e.g. cleft lip and palate) corroborate this finding, highlighting parental beliefs, emotions and values as key influences on the decision-making process $[10,19]$. It also has parallels with common-sense models of treatment adherence, particularly the Necessity-Concerns Framework [20], in which individuals are theorised to make rational choices about the treatments they accept based on weighing-up perceived need for treatment against perceived risks. Identifying relevant theoretical models is useful for the development of interventions to improve service provision.

Throughout their decision-making, parents identified additional information and emotional support needs. This finding confirms those of other studies $[2,9]$ in which patients and parents of children with intractable epilepsy describe receiving inadequate information regarding surgical treatment. It is already known that information helps to alleviate anxiety, and positively influences acceptance of treatment options including surgery $[1,7]$ and that physicians play a key role in influencing parents to accept surgery for their child with epilepsy through the provision of information [1, 2]. Since conducting this study, we have learnt that parents' perceived lack of support negates existing service provision (as described by professionals), including a dedicated CESS website and video, service-user involvement groups and direct telephone contact with the Epilepsy Nurse Specialist. It is clear then, that current family support mechanisms are either viewed as insufficient, falling short of parental need and expectation, or they require better promotion and active encouragement of family uptake. Research suggests that parents' present with a variety of information needs regarding the treatment of their chronically ill child and its expected outcomes [21]. Further evaluation of the timing, awareness, value and efficacy of existing parental support mechanisms is warranted.

Finally, studies show that parents who have been through the process of considering and accepting surgical intervention for their epileptic child would be willing to provide decisional support to other parents, as a way of 'giving back' to the service [9]. Parents in this study also suggested that they would be happy to share their experiences with others in similar situations. Facilitating this kind of peer-led intervention could provide a cost-effective way of enabling parents to be supported by other parents who are 'experts by experience' of the epilepsy surgery process.

\subsection{Limitations}

There are several limitations to this study. First, the sample size is small, so findings should be interpreted with caution. Second, as participants were recruited from one centre, their experiences may have limited transferability to other healthcare organisations and a multicentre study is recommended to test the generalisability of these findings. Nevertheless, our findings do confirm those of existing research, in particular a qualitative study conducted in the USA, exploring parent narratives of the journey to paediatric epilepsy surgery [9]. Third, all parents interviewed had accepted surgery for their child. A future study would be to include parents whose children were not deemed surgical candidates following assessment or those who were offered surgery but declined it. 
Furthermore, all parent participants described positive outcomes for their child following surgery, including reduced seizures. These positive health outcomes could have impacted on participants' responses and should be considered when appraising the findings. Finally, this study focused on parental experiences because of current drivers to increase access to and uptake of epilepsy surgery in younger children [4]. In practice, older children continue to be considered for epilepsy surgery. Subsequent studies should therefore include the perspectives of young people who are likely to contribute to the decision-making process alongside their parents.

\subsection{Implications for practice}

Parents and professionals identified a number of factors which contribute to difficulties in making decisions regarding treatment for a child's intractable epilepsy. Synthesising our findings with those of previous research $[1,8,9,13,17]$, we theorise important factors in the decision-making process to include the timing and presentation of surgery as a potential treatment option, incorporation of patient-related information within MDT meetings, knowledge and perceptions of intractable epilepsy and its treatments, access to decisional and emotional support. Addressing these issues could help to improve the uptake of surgical intervention. Our findings are being used to improve the paediatric epilepsy surgery pathway, including recommendations for:

- Earlier introduction/discussion of surgery as a potential treatment option for families to ensure decision-making is collaborative.

- Formalising families as part of the child's MDT by systematically incorporating patient/family perspectives within case presentations in MDT meetings.

- Providing additional and better promoted information to families in a variety of formats (e.g. information packs, videos, email address for submitting questions about the surgical procedure, from which a list of parent generated FAQ can be produced).

- Providing parental peer support by facilitating access to other parents who are deemed 'experts by experience'.

\section{Conclusion}

Parents in this study suggested that they can be supported to make decisions regarding paediatric epilepsy surgery by incorporating them into discussions regarding epilepsy surgery as a potential treatment option earlier in the process, and by providing additional information and access to other parents with similar experiences. Findings support those of previous research and could be used to improve uptake of surgical intervention in those children for whom it is an option and would be of benefit.

\section{References}

1. Erba, G., et al., Acceptance of epilepsy surgery in the pediatric age - What the parents think and what the doctors can do. Epilepsy \& Behavior, 2013. 29: p. 112-120.

2. Swarztrauber, K., S. Dewar, and J. Engel, J. Patient attitudes about treatments for intractable epolepsy Epilepsy \& Behavior, 2003. 4(1): p. 19-25.

3. Hakimi, A.S., et al., A survey of neurologists' views on epilepsy surgery and medically refractory epilepsy. Epilepsy \& Behavior, 2008. 13(1): p. 96-101. 
4. NHS England, 2013/14 NHS Standard Contract For Children's Epilepsy Surgery Service (CESS). 2013.

5. Harvey, S., et al., Defining the spectrum of international practice in pediatric epilepsy surgery patients. Epilepsia, 2008. 49: p. 146-155.

6. Ibrahim, G.M., et al., Ethical issues in surgical decision making concerning children with medically intractable epilepsy. Epilepsy \& Behavior, 2011. 22: p. 154-157.

7. Beresford, B. and P. Sloper, Understanding the dynamics of decision-making and choice: A scoping study of key psychological theories to inform the design and analysis of the panel study. 2008, University of York: Social Policy Research Unit

8. Dewar, S.R. and H.C. Pieters, Percpeitons of epilepsy surgery: A systematic review and explanatory model of decision-making. Epilepsy \& Behavior, 2015. 44: p. 171-178.

9. Baca, C.B., et al., A journey around the world": Parent narratives of the journey to pediatric resective epilepsy surgery and beyond. Epilepsia, 2015. 56(6): p. 822-832.

10. Lipstein, E.A., W.B. Brinkman, and M.T. Britto, What Is Known about Parents' Treatment Decisions? A Narrative Review of Pediatric Decision Making. Medical Decision Making, 2012. 32: p. 246-258.

11. Erba, G., et al., Barriers toward epilepsy surgery. A survey among practicing neurologists. . Epilepsia 2012. 53(1): p. 35-43.

12. Kumlien, E. and P. Mattsson, Attitudes towards epilepsy surgery: a nationwide survey among Swedish neurologists. Seizure, 2010. 19(4): p. 253-255.

13. Kidger, J., et al., Clinical decision-making in a multidisciplinary gynaecological cancer team: a qualitative study. Gynaecological Oncology, 2009. 116: p. 511-517.

14. Lanceley, A., et al., Influences on multidisciplinary team decision-making. International Journal of Gynecological Cancer, 2008. 18: p. 215-222.

15. Spradley, J.P., Participant observation. . 1980, New York:: Holt, Reinhart \& Winston.

16. Braun, V. and V. Clarke, Using thematic analysis in psychology. Qualitative Research in Psychology, 2006. 3: p. 77-101.

17. Choi, H., et al., Temporal lobe epilepsy surgery: What do patients want to know? Epilepsy \& Behavior, 2011. 22: p. 479-482.

18. Leventhal, H., D. Meyer, and D. Nerenz, The common-sense representations of illness danger, in Medical Psychology, S. Rachman, Editor. 1980, Pergamon: New York. p. 7-30.

19. Nelson, P. A., et al., 'Doing the "Right" Thing': How parents experience and manage decisionmaking for children's 'Normalising' surgeries. Social Science \& Medicine, 2012. 74: p.796804.

20. Horne, R. and J. Weinman, Patients' beliefs about prescribed medicines and their role in adherence to treatment in chronic physical illness. Journal of Psychosomatic Research, 1999. 47(6): p. 555-567.

21. Hummelinck, A. and K. Pollock, Parents' information needs about the treatment of their chronically ill child: A qualitative study. Patient Education and Counseling, 2006. 62: p. 228234. 